\title{
Gender Difference in the Association Between Uric Acid and Atrial Fibrillation
}

\author{
Masanari Kuwabara, MD, $\mathrm{PhD}$; Ichiro Hisatome, $\mathrm{MD}, \mathrm{PhD}$
}

$\mathbf{T}$ The association between hyperuricemia and atrial fibrillation (AF) is well established, ${ }^{1}$ but not whether the relationship is causal or not. ${ }^{2}$ The first report regarding the relationship between serum uric acid (SUA) and permanent AF was from Greece and published in $2010 .{ }^{3}$ So far, more than 100 articles regarding this issue have been published. Some large-scale cohort studies report that hyperuricemia is associated with the new development of $\mathrm{AF},{ }^{4,5}$ and meta-analysis also has shown a positive relationship between increased SUA and AF. ${ }^{6}$ Moreover, a high SUA level is a risk for AF recurrence after catheter ablation $^{7}$ and left atrial thrombus or spontaneous echo contrast detected by echocardiography. ${ }^{8}$ However, the SUA level is easily influenced by many other factors such as gender, meals, drink (e.g., alcohol, fructose), lifestyle, obesity, metabolic syndrome, hypertension, diabetes, dyslipidemia, chronic kidney disease, and use of medications such as diuretics. ${ }^{9}$ When any UA study is conducted, these

\section{Article p 156}

factors and comorbidities have to be taken into account. Our previous study of subjects without commodities, such as hypertension, diabetes mellitus, dyslipidemia, chronic kidney disease, and current medications for hyperuricemia and/or gout showed that hyperuricemia is an independent competing risk factor for AF in both men and women. ${ }^{10}$ However, we cannot tell whether UA alone induces AF, because there has not been a large-scale intervention study to confirm whether SUA-lowering therapy is effective for AF prevention.

The article by Kawasoe et al in this issue of the Journal is well written and designed to evaluate the relationship between SUA and new onset AF. ${ }^{11}$ The study included 111,566 subjects with data from annual health checkups, and all the analyses were stratified by gender to investigate
\end{abstract}

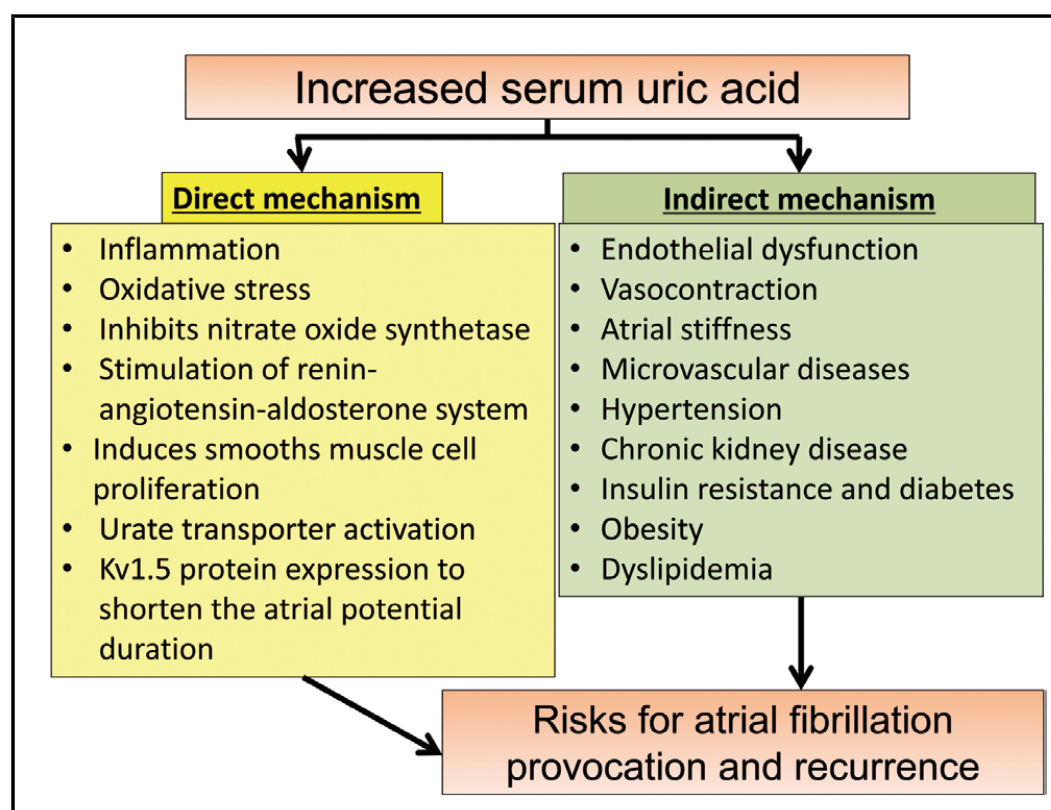

Figure 1. Possible mechanisms of serum uric acid-induced atrial fibrillation.

The opinions expressed in this article are not necessarily those of the editors or of the Japanese Circulation Society.

Received November 16, 2018; accepted November 18, 2018; J-STAGE Advance Publication released online November 30,2018

Department of Cardiology and Intensive Care Unit, Toranomon Hospital, Tokyo (M.K.); Institute of Regenerative Medicine and Biofunction, Tottori University Graduate School of Medical Science, Yonago (I.H.), Japan

Mailing address: Ichiro Hisatome, MD, PhD, Institute of Regenerative Medicine and Biofunction, Tottori University Graduate School of Medical Science, 86 Nishi-machi, Yonago 683-8503, Japan. E-mail: hisatome@tottori-u.ac.jp

ISSN-1346-9843 All rights are reserved to the Japanese Circulation Society. For permissions, please e-mail: cj@j-circ.or.jp 


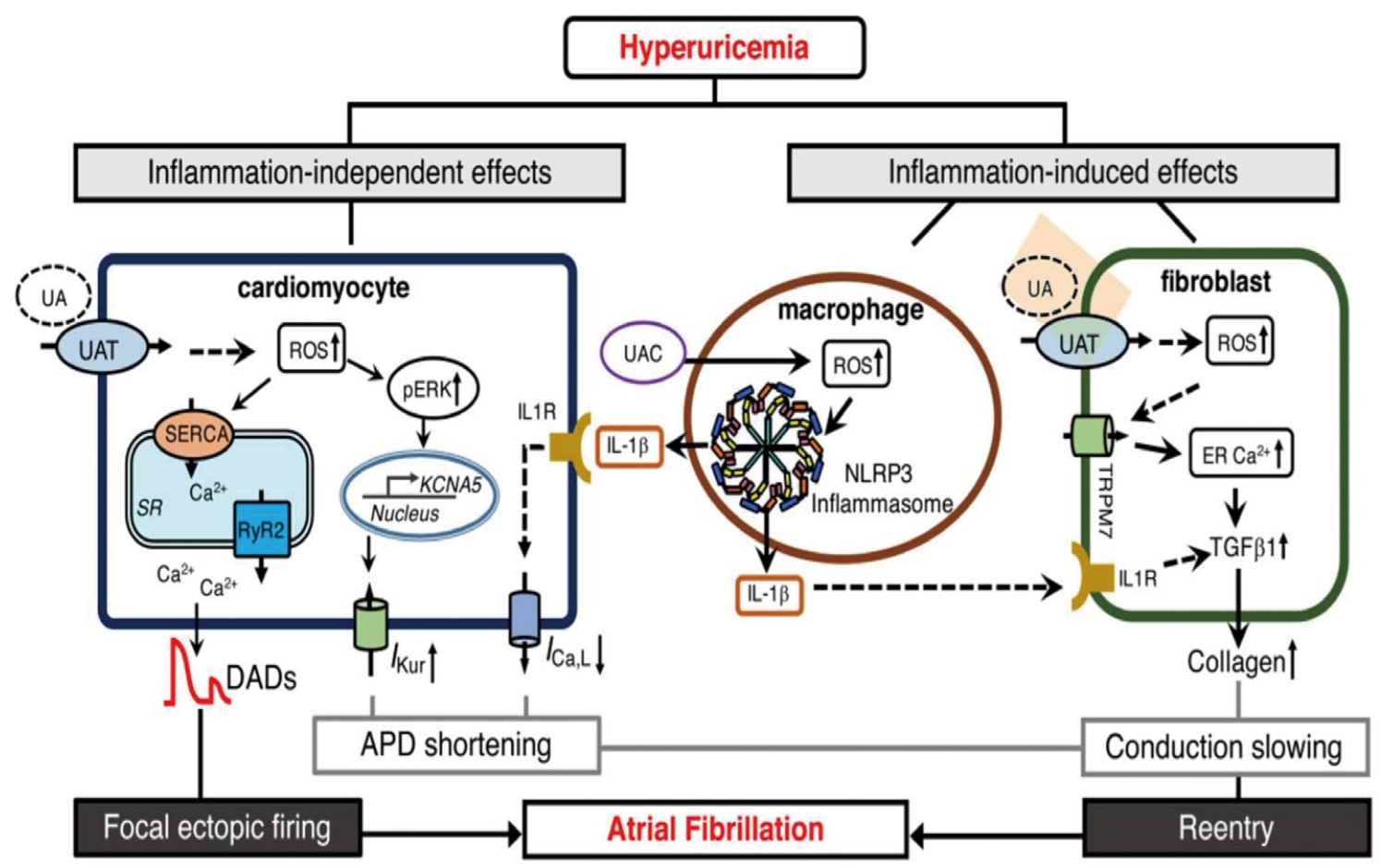

Figure 2. Putative mechanisms of hyperuricemia-induced atrial fibrillation. ${ }^{12}$ APD, action potential duration; DADs, delayed afterdepolarizations; ER, endoplasmic reticulum; IL, interleukin; NLRP3, NACHT, LRR and PYD domain-containing protein 3; pERK, phosphorylated (activated) extracellular signal-regulated kinase; ROS, reactive oxygen species; RyR2, ryanodine receptor type-2; SERCA, sarcoplasmic reticulum (SR) Ca ${ }^{2+}$ ATPase; TGF, transforming growth factor; UA, uric acid. Solid lines indicate proven mechanisms, dashed lines indicate putative pathways. For further details see text. (Reproduced with permission from Li N, et al. ${ }^{\mathbf{1 2}}$ )

the predictive power of SUA for new-onset AF over 4.1 years after adjustments for body mass index, creatinine, smoking and drinking status, hypertension, diabetes, and dyslipidemia. The results showed that the 3rd tertile of SUA level $(>4.9 \mathrm{mg} / \mathrm{dL})$ was a significantly higher risk than the 1 st tertile $(<3.9 \mathrm{mg} / \mathrm{dL})$ in women (hazard ratio $(\mathrm{HR})$, $1.74 ; 95 \%$ confidence interval, $1.15-2.70 ; \mathrm{P}=0.008)$, whereas there were no differences among tertiles in men $(<5.4$, $5.5-6.4$, and $>6.5 \mathrm{mg} / \mathrm{dL}$ ). Moreover, Kawasoe et al show that the group with initially-increased SUA ( $\triangle$ SUA $>0.3 \mathrm{mg} / \mathrm{dL}$ ) had a significantly higher rate of new-onset $\mathrm{AF}$ than the group with unchanged SUA $(\Delta \mathrm{SUA}< \pm 0.2 \mathrm{mg} / \mathrm{dL})$ in the 3rd tertile of baseline SUA in both women and men. Importantly, this study showed that the association between SUA level and new-onset AF may differ between men and women. Moreover, the association between initial increase of SUA and new-onset AF was detected only in the hyperuricemic state (the 3rd tertile of SUA ${ }^{\mathbf{1 1}}$ ).

How hyperuricemia induces AF is attributed to 2 possible mechanisms: direct and indirect (Figure 1). Hyperuricemia is a well-known risk factor for hypertension and chronic kidney diseases, which are also risks for AF. From this viewpoint, hyperuricemia could be a risk for AF indirectly. Besides, UA itself directly induces AF by both inflammation-induced and inflammation-independent effects (Figure 2). ${ }^{12}$ Urate crystals induce inflammation by activation of the NACHT, LRR and PYD domain-containing protein 3 (NLRP3) inflammasome in macrophages. ${ }^{12} \mathrm{We}$ also reported another mechanism involving soluble UA through the urate transporter to induce AF. Activation of the Kv1.5 channel is one of the main factors for the provocation and recurrence of AF. The accumulation of intracellular UA via activation of urate transporters enhances Kv1.5 protein expression to shorten the atrial action potential duration, ${ }^{13}$ which contributes to the reentry circuit for AF.

Pertinently, and interestingly, Kawasoe et al demonstrate a gender difference regarding the effects of SUA on newonset AF. The gender difference observed in this study is consistent with a previous study by Suzuki et al, ${ }^{14}$ which could be explained by the unique feature of SUA levels in women associated with aging. Female hormones such as estrogen decrease the SUA level; thus, the SUA level increases after menopause. Interestingly, the expression of urate transporters such as SLC2A9 is known to be regulated by estrogen. ${ }^{15}$ Thus, the gender-dependent effects of SUA on new-onset AF observed in Kawasoe et al's article may be attributed to the estrogen-induced modification of the expression level of atrial urate transporters, which could facilitate the accumulation of intracellular UA via enhanced expression of urate transporters by female hormones.

We have to pay attention to aging as the most important risk factor for AF ${ }^{10}$ However, not only the prevalence of AF but also the SUA level increases with aging after menopause in women. Although this study by Kawasoe et al adjusted age as well as other comorbidities in the multivariate analysis, it could be difficult to adjust these cofactors 
completely.

There are some experimental findings on why hyperuricemia induces AF,12 but little clinical evidence showing that SUA-lowering therapy could prevent AF provocation. We need further high-quality clinical intervention studies to clarify whether SUA-lowering therapy for hyperuricemic patients can prevent new-onset AF.

\section{Name of Grant}

None.

\section{References}

1. Maharani N, Kuwabara M, Hisatome I. Hyperuricemia and atrial fibrillation. Int Heart J 2016; 57: 395-399.

2. Watanabe E. Uric acid and atrial fibrillation: Cause or other association? Circ J 2012; 76: 584-585.

3. Letsas KP, Korantzopoulos P, Filippatos GS, Mihas CC Markou V, Gavrielatos G, et al. Uric acid elevation in atrial fibrillation. Hellenic J Cardiol 2010; 51: 209-213.

4. Chao TF, Hung CL, Chen SJ, Wang KL, Chen TJ, Lin YJ, et al. The association between hyperuricemia, left atrial size and newonset atrial fibrillation. Int J Cardiol 2013; 168: 4027-4032.

5. Nyrnes A, Toft I, Njolstad I, Mathiesen EB, Wilsgaard T, Hansen JB, et al. Uric acid is associated with future atrial fibrillation: An 11-year follow-up of 6308 men and women - the Tromso Study. Europace 2014; 16: 320-326.

6. Tamariz L, Hernandez F, Bush A, Palacio A, Hare JM. Association between serum uric acid and atrial fibrillation: A systematic review and meta-analysis. Heart Rhythm 2014; 11: 1102-1108.
7. Zhao J, Liu T, Korantzopoulos P, Letsas KP, Zhang E, Yang Y, et al. Association between serum uric acid and atrial fibrillation recurrence following catheter ablation: A meta-analysis. Int $J$ Cardiol 2016; 204: 103-105.

8. Zhang EY, Kou L, Li M, Ng CY, Zhao JP, Li GP, et al. High uric acid level predicts left atrial thrombus or spontaneous echo contrast detected by transesophageal echocardiography: Meta-analysis and systematic review. Chronic Dis Transl Med 2016; 2: $27-33$.

9. Kuwabara M. Hyperuricemia, cardiovascular disease, and hypertension. Pulse (Basel) 2016; 3: 242-252.

10. Kuwabara M, Niwa K, Nishihara S, Nishi Y, Takahashi O, Kario K, et al. Hyperuricemia is an independent competing risk factor for atrial fibrillation. Int J Cardiol 2017; 231: 137-142.

11. Kawasoe S, Kubozono T, Yoshifuku S, Ojima S, Miyata M, Miyahara $\mathrm{H}$, et al. Uric acid level and new-onset atrial fibrillation in the Japanese general population: Longitudinal study. Circ J 2019; 83: $156-163$.

12. Li N, Dobrev D. Hyperuricemia: A causal player or a bystander linking inflammatory signaling and atrial fibrillation? Int J Cardiol 2017; 231: $177-178$.

13. Maharani N, Ting YK, Cheng J, Hasegawa A, Kurata Y, Li P, et al. Molecular mechanisms underlying urate-induced enhancement of Kv1.5 channel expression in HL-1 atrial myocytes. Circ J 2015; 79: 2659-2668.

14. Suzuki S, Sagara K, Otsuka T, Matsuno S, Funada R, Uejima $\mathrm{T}$, et al. Gender-specific relationship between serum uric acid level and atrial fibrillation prevalence. Circ J 2012; 76: 607-611.

15. Doring A, Gieger C, Mehta D, Gohlke H, Prokisch H, Coassin $\mathrm{S}$, et al. SLC2A9 influences uric acid concentrations with pronounced sex-specific effects. Nat Genet 2008; 40: 430-436. 